\title{
Effect of Oral Hypoglycaemic Drugs on Glucose Tolerance and Insulin Secretion in Borderline Diabetic Patients*
}

\author{
L. Papoz ${ }^{1}$, D. Job ${ }^{1}$, E. Eschwege ${ }^{1}$, J. P. Aboulker ${ }^{1}$, J. Cubeau ${ }^{2}$, G. Pequignot ${ }^{2}$, M. Rathery ${ }^{3}$, and G. Rosselin ${ }^{4}$ \\ ${ }^{1}$ Unité de Recherches Statistiques INSERM, Villejuif, ${ }^{2}$ Section Nutrition, INSERM, Le Vesinet, ${ }^{3}$ Centre de Dépistage du Diabète, Hôtel- \\ Dieu, Paris, and ${ }^{4}$ Unité de Recherches de Diabétologie et d'Etudes des Hormones Protéiques INSERM, Hôpital St Antoine, Paris, France
}

Summary. A double blind controlled clinical trial was undertaken to test the effectiveness of oral hypoglycaemic drugs in improving blood glucose and plasma insulin levels of borderline diabetic patients. Between 1969 and 1971, 120 men aged 25 to 55 with borderline impairment of glucose tolerance according to standard criteria at 2 successive oral glucose tolerance tests entered the study. They were randomized into 4 groups according to treatment: dimethylbiguanide + glibenclamide $(\mathrm{n}=29)$, placebo $\mathrm{B}+$ glibenclamide $(\mathrm{n}=28)$, placebo $\mathrm{S}+$ dimethylbiguanide $(\mathrm{n}=30)$, placebo $\mathrm{B}+\mathrm{S}(\mathrm{n}=$ 33). In each group drugs were taken twice a day before breakfast and lunch at a total dosage of $1.7 \mathrm{~g} /$ day dimethylbiguanide and/or $4 \mathrm{mg} /$ day glibenclamide. Treatment was stopped after 2 years. Patients returned 2 months after entry into the trial, then every 4 months for 2 years. Treatment was continued up to each oral glucose tolerance test except before the last test (drugs stopped 15 days before). 29 patients weighed $20 \%$ or more over their ideal body weight and 23 between $10 \%$ and $20 \%$. After a dietary survey, these men were subjected to a total colorie restricted diet according to their excess weight. Results indicate that during the 2 years of treatment, no significant lasting effect of the biguanide on blood glucose and plasma insulin levels was detectable. During the oral glucose tolerance test at 14 months in the groups receiving sulphonylurea a significant decrease of blood glucose levels was observed at 0,180 and $240 \mathrm{~min}$. Glibenclamide had no effect on weight reduction while biguanide administration was accompanied by a significant weight reduction.

* Parts of this paper were presented at the 10th meeting of the EASD in Jerusalem and published in abstract form in Diabetologia 10, 363 (1974)
Key words: Randomized clinical trial, borderline diabetes, oral hypoglycaemic drugs, glucose tolerance, insulin secretion, dimethylbiguanide, sulphonylurea.

Prospective studies in man [1-3] and animals [4-6] support the concept that optimal control of blood glucose level delays the development of microvascular lesions in overt diabetes. Lowering blood glucose levels in borderline diabetic patients, then, appears a logical aim of treatment in order to achieve early prevention of degenerative vascular changes. We have therefore investigated the effectiveness of oral hypoglycaemic drugs on the glucose and insulin responses to an oral glucose load in patients with borderline impairment of glucose tolerance [7], in a controlled clinical trial.

\section{Material and Methods}

\section{Oral Glucose Tolerance Test}

Because of the variability in carbohydrate tolerance at successive examinations [8-12], a two step procedure was employed to test eligibility of patients:

1. At the first test venous blood glucose concentration fasting was measured, $\left(\mathrm{BG}_{\mathrm{o}}\right)$ and $2 \mathrm{~h}\left(\mathrm{BG}_{2}\right)$ after a $75 \mathrm{~g}$ oral glucose load, by a glucose - oxidase method [13]. The subjects were classified according to the European Diabetes Epidemiology Study Group (EDESG) criteria [14-15]: when $\mathrm{BG}_{\mathrm{o}}$ was lower than $100 \mathrm{mg}$ / $100 \mathrm{ml}$ and $\mathrm{BG}_{2}$ lower than $120 \mathrm{mg} / 100 \mathrm{ml}$ the subject was considered normal, when $\mathrm{BG}_{\mathrm{o}}$ was equal to $130 \mathrm{mg} / 100 \mathrm{ml}$ or more and $\mathrm{BG}_{2}$ equal to $150 \mathrm{mg} / 100 \mathrm{ml}$ or more, the subject was considered diabetic. All remaining subjects were classified borderline and submitted to the second test.

2. The second test was performed 8 to 15 days later under standard conditions [16] with, in particular, dietary preparation 
Table 1. Distribution of 120 eligible subjects according to 6 different criteria [20] applied to the $5 \mathrm{~h}$ OGTT results

The subjects are recognized as:

\begin{tabular}{llll}
\hline Normal & & Diabetic & Borderline \\
\cline { 1 - 1 } $\begin{array}{l}\text { No of } \\
\text { patients } \%\end{array}$ & $\begin{array}{l}\text { No of } \\
\text { patients } \%\end{array}$ & $\begin{array}{l}\text { No of } \\
\text { patients } \%\end{array}$ \\
\hline
\end{tabular}

According to the

criteria of:

Fajans, Conn [35]

Wilkerson [18]

BDA [19]

UGDP [31]

WHO [36]

EGSED [14-15]

$\begin{array}{rcc}40 & 33 & 80 \\ 100 & 83 & 20 \\ 31 & 26 & 89 \\ 36 & 30 & 84 \\ 27 & 23 & 64 \\ 0 & - & 13^{\mathrm{a}} \\ 0 & - & 8\end{array}$

$\begin{array}{rll}67 & - & - \\ 17 & - & - \\ 74 & - & - \\ 70 & - & - \\ 53 & 29 & 24 \\ 11 & 107 & 89 \\ 7 & 112^{\mathrm{b}} & 93\end{array}$

a These subjects were classified as borderline at the first test by the EDESG criteria

b Patients who were not recognized as normal or diabetic by all criteria were classified as borderline

including $250 \mathrm{~g}$ of carbohydrates for the three days before the test. Blood glucose levels were determined fasting and at $15,30,60$, $120,180,240$ and $300 \mathrm{~min}$ after an oral glucose load. Two limits $l_{1}$ and $\mathrm{l}_{2}$ were defined for the blood glucose levels measured at zero, 30,60 and 120 min post glucose load $\left(\mathrm{BG}_{\mathrm{o}}, \mathrm{BG}_{30}, \mathrm{BG}_{60}, \mathrm{BG}_{120}\right)$. Below the lower limit $\mathbf{l}_{1}$, blood glucose values were considered normal; on the upper limit $l_{2}$ or above, blood glucose values were considered diabetic. The chosen values were for $l_{1}$ respectively $100,160,160$ and $120 \mathrm{mg} / 100 \mathrm{ml}$ and for $l_{2}$ respectively 130,220 , 220 and $150 \mathrm{mg} / 100 \mathrm{ml}$, as recommended by the EDESG [14].

According to these criteria, the subjects were classified as follows: all subjects with normal responses at the four times were excluded as well as the subjects with both $\mathrm{BG}_{0}$ and $\mathrm{BG}_{120}$ diabetic responses. Among the remainder, eligible subjects were either those with $\mathrm{BG}_{120}$ equal to or greater than $120 \mathrm{mg} / 100 \mathrm{ml}$ or else those with $\mathrm{BG}_{\mathrm{o}}, \mathrm{BG}_{30}$ and $\mathrm{BG}_{60}$ all equal to or greater than their respective limits $l_{1}$.

These criteria were established prior to the beginning of the trial according to the experience of 7 diabetologists in order to exclude any subject for whom they judged there was no possible choice between treatment and placebo [17].

\section{Patients}

One hundred and twenty patients were recruited from among the male population spontaneously asking medical advice in a screening centre for Diabetes at the Hotel Dieu Hospital in Paris, between March 1969 and October 1971. They were 25 to 55 years old, free from any other apparent clinical disease, and taking no drugs. $29(25 \%)$ of them were $20 \%$ or more over their ideal body weight and $23(19 \%)$ between $10 \%$ and $20 \%$. Thirty four patients ( 24 during the first year, 10 during the second year of the study) were lost to follow-up; they came equally from the four different treatment groups and exhibited similar baseline characteristics to the follow-up patients. Their removal from the trial did not introduce any bias into the study.

To judge the inclusion criteria previously defined the 120 subjects selected for the trial were classified according to 6 different international criteria regarding their $5 \mathrm{~h}$ OGTT results (Table 1). In the study sample, $83 \%$ were normal according to the Wilkerson point system [18] whereas only $26 \%$ were normal according to the
British Diabetic Association criteria [19]. None of the subjects would be recognized as normal by all criteria, while $7 \%$ of thern would be universally recognized as diabetic. These results show the discrepancy between the different criteria as emphasized in another study [20].

\section{Allocation to Treatment}

Based upon sequence of entry to the study, patients in each group were assigned a trial number. This determined the treatment they received, but the trial number gave no indication of the treatment. Randomization with equal numbers of patients in each treatment group was carried out.

\section{Treatment}

Four treatment groups were studied:

1. Sulphonylurea-treated group (S group) who received $2 \mathrm{mg}$ of Glibenclamide and a biguanide placebo before breakfast and before lunch.

2. The biguanide-treated group (B) received $0.85 \mathrm{~g}$ of dimethylbiguanide and a sulphonylurea placebo before breakfast and before lunch.

3. Combined therapy group $(B+S)$ who received both drugs in the above doses.

4. The placebo group $(P)$ who received a sulphonylurea placebo and a biguanide placebo.

Tablets were taken every day for the 2 years of the trial. None of the patients under study exhibited symptoms of intolerance requiring a change of therapy. Fourteen patients, equally distributed in the four groups, admitted to having stopped their drug intake for 1 month or less.

All patients were regularly questioned on their dietary habits. Overweight patients were prescribed calorie restriction in order to approach their ideal body weight [21]. The diet was aimed at restricting firstly, alcohol consumption, then sweets and pastries, then extra fats, such as butter and oil, and finally bread if necessary. In all cases, the balance between the different nutrients was respected.

\section{Data Collection}

Every four months, clinical and biochemical examinations were performed. In practice, none of the patients exhibited any pathological symptom during the survey. Oral glucose tolerance tests were performed 2,14 and 26 months after the baseline test (i. e. 2 nd test for eligibility) as defined above. At 2 and 14 months, the patients received their tablets before the oral glucose tolerance test. Then the treatments were stopped 15 days before the 26 months test. Besides blood glucose measurements, plasma insulin levels were estimated by radioimmunoassay [22].

\section{Statistical Analysis}

The study was designed for two way analysis of variance [23]. Thus, the inclusion of the four possible combinations of treatments into the study allowed us to test if:

1. the effect of biguanides was the same whether sulphonylureas were given at the same time or not,

2. the effect of sulphonylureas was the same whether biguanides were given at the same time or not (test of interaction).

Absence of significant interaction allowed the biguanide effect to be tested by the comparison of both group $B$ with group $P$, and of group B $+S$ with group S: and, similarly, to test the effect of the 
Table 2. Baseline characteristics in the 4 groups of treatment: placebo (P), biguanide (B), sulphonylurea (S) and combined therapy $(B+S)$. The values represent mean $\pm S E M$ and the range is in parenthesis

\begin{tabular}{lcccc}
\hline Group & $\mathrm{n}$ & Age (a) & Weight (kg) & Overweight (\%) \\
\hline $\mathrm{P}$ & 33 & $45 \pm 1$ & $79 \pm 2$ & $14 \pm 2$ \\
& & $(25-54)$ & $(65-118)$ & $(-5-47)$ \\
$\mathrm{B}$ & 30 & $44 \pm 1$ & $74 \pm 2$ & $8 \pm 2$ \\
& & $(26-53)$ & $(63-91)$ & $(0-43)$ \\
$\mathrm{S}$ & 28 & $43 \pm 2$ & $75 \pm 2$ & $9 \pm 3$ \\
& & $(25-54)$ & $(56-99)$ & $(-11-50)$ \\
$\mathrm{B}+\mathrm{S}$ & 29 & $44 \pm 1$ & $78 \pm 3$ & $14 \pm 3$ \\
& & $(29-54)$ & $(54-106)$ & $(-13-85)$ \\
\hline
\end{tabular}

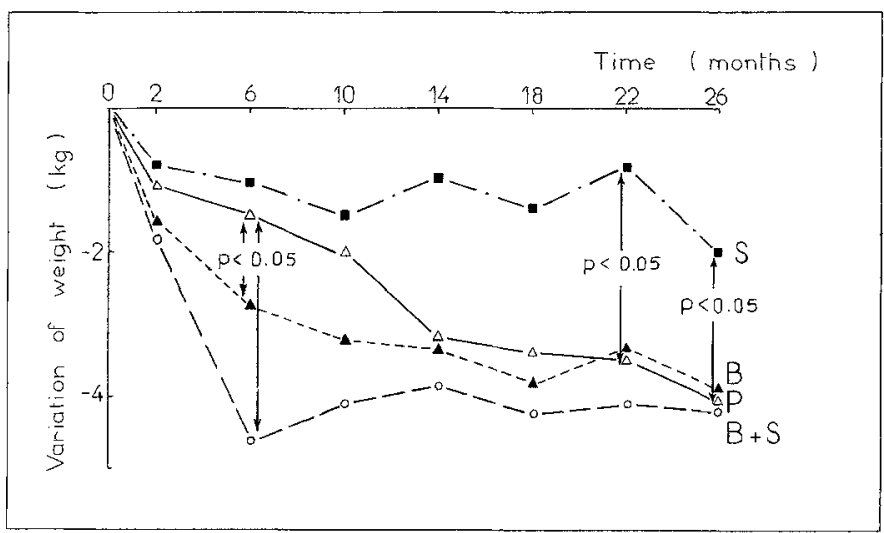

Fig. 1. Changes in weight from baseline in the 4 groups: placebo $(\mathrm{P})$, biguanide $(\mathrm{B})$, sulphonylurea $(\mathrm{S})$ and combined therapy $(\mathrm{B}+$ S)

Table 3. Baseline blood glucose and plasma insulin values in the 4 groups of treatment: placebo (P), biguanide (B), sulphonylurea (S) and combined therapy $(B+S)$. The values represent mean $\pm S E M$ and the range is in parenthesis

\begin{tabular}{|c|c|c|c|c|c|c|c|c|c|}
\hline \multirow[t]{2}{*}{ Group } & \multirow[t]{2}{*}{$\mathrm{n}$} & \multicolumn{8}{|c|}{ Time (min) after glucose load } \\
\hline & & 0 & 15 & 30 & 60 & 120 & 180 & 240 & 300 \\
\hline \multicolumn{10}{|c|}{ a) Blood giucose $(\mathrm{mg} / 100 \mathrm{ml})$} \\
\hline $\mathrm{P}$ & 33 & $\begin{array}{l}103 \pm 2 \\
(79-121)\end{array}$ & $\begin{array}{c}130 \pm 3 \\
(101-176)\end{array}$ & $\begin{array}{c}172 \pm 4 \\
(117-228)\end{array}$ & $\begin{array}{c}199 \pm 5 \\
(150-282)\end{array}$ & $\begin{array}{l}136 \pm 6 \\
(67-210)\end{array}$ & $\begin{array}{c}85 \pm 5 \\
(58-168)\end{array}$ & $\begin{array}{c}76 \pm 2 \\
(55-103)\end{array}$ & $\begin{array}{c}79 \pm 2 \\
(65-98)\end{array}$ \\
\hline $\mathrm{B}$ & 30 & $\begin{array}{l}108 \pm 2 \\
(90-126)\end{array}$ & $\begin{array}{c}134 \pm 4 \\
(102-176)\end{array}$ & $\begin{array}{c}170 \pm 5 \\
(122-228)\end{array}$ & $\begin{array}{c}190 \pm 7 \\
(106-263)\end{array}$ & $\begin{array}{l}133 \pm 5 \\
(79-197)\end{array}$ & $\begin{array}{l}82 \pm 5 \\
(49-160)\end{array}$ & $\begin{array}{l}77 \pm 2 \\
(56-101)\end{array}$ & $\begin{array}{l}82 \pm 2 \\
(62-100)\end{array}$ \\
\hline $\mathrm{S}$ & 28 & $\begin{array}{l}101 \pm 2 \\
(84-134)\end{array}$ & $\begin{array}{l}121 \pm 4 \\
(90-159)\end{array}$ & $\begin{array}{c}156 \pm 5 \\
(120-222)\end{array}$ & $\begin{array}{c}184 \pm 7 \\
(112-250)\end{array}$ & $\begin{array}{l}143 \pm 6 \\
(69-224)\end{array}$ & $\begin{array}{c}82 \pm 4 \\
(56-153)\end{array}$ & $\begin{array}{c}73 \pm 2 \\
(52-105)\end{array}$ & $\begin{array}{l}79 \pm 2 \\
(56-102)\end{array}$ \\
\hline $\mathrm{B}+\mathrm{S}$ & 29 & $\begin{array}{l}104 \pm 2 \\
(76-124)\end{array}$ & $\begin{array}{l}129 \pm 3 \\
(96-175)\end{array}$ & $\begin{array}{c}167 \pm 5 \\
(112-244)\end{array}$ & $\begin{array}{c}198 \pm 6 \\
(121-300)\end{array}$ & $\begin{array}{l}133 \pm 6 \\
(60-214)\end{array}$ & $\begin{array}{c}83 \pm 5 \\
(49-133)\end{array}$ & $\begin{array}{c}76 \pm 3 \\
(50-124)\end{array}$ & $\begin{array}{c}79 \pm 2 \\
(51-98)\end{array}$ \\
\hline \multicolumn{10}{|c|}{ b) Plasma insulin $(\mu \mathrm{U} / \mathrm{ml})$} \\
\hline $\mathrm{P}$ & 31 & $\begin{array}{l}16 \pm 2 \\
(2-40)\end{array}$ & $\begin{array}{c}35 \pm 4 \\
(13-72)\end{array}$ & $\begin{array}{c}56 \pm 6 \\
(19-136)\end{array}$ & $\begin{array}{c}85 \pm 10 \\
(20-340)\end{array}$ & $\begin{array}{c}75 \pm 13 \\
(10-380)\end{array}$ & $\begin{array}{l}26 \pm 4 \\
(4-116)\end{array}$ & $\begin{array}{l}15 \pm 1 \\
(4-33)\end{array}$ & $\begin{array}{l}12 \pm 1 \\
(5-25)\end{array}$ \\
\hline B & 29 & $\begin{array}{l}14 \pm 1 \\
(6-30)\end{array}$ & $\begin{array}{c}45 \pm 4 \\
(10-86)\end{array}$ & $\begin{array}{c}64 \pm 7 \\
(10-144)\end{array}$ & $\begin{array}{l}99 \pm 8 \\
(16-200)\end{array}$ & $\begin{array}{l}71 \pm 7 \\
(16-130)\end{array}$ & $\begin{array}{l}28 \pm 5 \\
(5-133)\end{array}$ & $\begin{array}{l}15 \pm 2 \\
(3-33)\end{array}$ & $\begin{array}{l}13 \pm 1 \\
(3-34)\end{array}$ \\
\hline$S$ & 28 & $\begin{array}{l}16 \pm 2 \\
(4-66)\end{array}$ & $\begin{array}{c}30 \pm 4 \\
(10-112)\end{array}$ & $\begin{array}{c}48 \pm 7 \\
(14-136)\end{array}$ & $\begin{array}{l}74 \pm 8 \\
(20-156)\end{array}$ & $\begin{array}{c}73 \pm 7 \\
(28-150)\end{array}$ & $\begin{array}{l}27 \pm 5 \\
(6-134)\end{array}$ & $\begin{array}{l}14 \pm 2 \\
(3-40)\end{array}$ & $\begin{array}{l}12 \pm 1 \\
(2-25)\end{array}$ \\
\hline$B+S$ & 28 & $\begin{array}{l}16 \pm 2 \\
(2-42)\end{array}$ & $\begin{array}{l}35 \pm 3 \\
(2-73)\end{array}$ & $\begin{array}{l}51 \pm 5 \\
(21-117)\end{array}$ & $\begin{array}{c}87 \pm 13 \\
(17-200)\end{array}$ & $\begin{array}{c}95 \pm 5 \\
(23-400)\end{array}$ & $\begin{array}{l}33 \pm 5 \\
(11-116)\end{array}$ & $\begin{array}{l}18 \pm 2 \\
(2-58)\end{array}$ & $\begin{array}{l}14 \pm 1 \\
(2-26)\end{array}$ \\
\hline
\end{tabular}

sulphonylureas by comparison of group $\mathrm{S}$ with group $\mathrm{P}$ and of group $\mathrm{B}+\mathrm{S}$ with group $\mathrm{B}$.

Finally, classical analysis of variance provided a test for the effect of biguanide and sulphonylurea which involved all the patients under study in each comparison. This achieved a more efficient method of analysis than the usual comparisons of means between groups.

\section{Results}

\section{Baseline Characteristics}

Comparison of the four groups was performed for each baseline characteristic (clinical table 2; biochemical table 3 ). No significant difference between the 4 groups was observed; this indicates the effectiveness of the randomisation.

\section{Body Weight}

Mean changes in weight are shown for the 4 groups in figure 1. Weight reduction was observed in each group, placebo group included. All these reductions were significantly different from zero by Student's t-test. Moreover, the comparisons with the placebo group showed at 6 months a greater weight loss in the 2 groups receiving biguanide. The weight loss was smaller in the $\mathrm{S}$ group than in the $\mathrm{P}$ group, differences reaching a significant level at 22 and 26 months.

Results of the two way analysis of variance are shown in Table 4. At 6 months and afterwards body weight was significantly lower in the $\mathrm{B}$ and $\mathrm{B}+\mathrm{S}$ groups than in the $\mathrm{P}$ and $\mathrm{S}$ groups. No significant 
Table 4. Effect of biguanide (B) and sulphonylurea (S) on body weight (kg). Group "with B" includes B and B + S - group "without B" includes $\mathrm{P}$ and $\mathrm{S}$. Group "with $\mathrm{S}$ " includes $\mathrm{S}$ and $\mathrm{B}+\mathrm{S}-$ group "without $\mathrm{S}$ " includes $\mathrm{P}$ and B. Values represent mean $\pm \mathrm{SEM}$; NS $=$ Differences not significant. Number of subjects is indicated in parenthesis

\begin{tabular}{|c|c|c|c|c|c|c|c|}
\hline \multirow[t]{2}{*}{ Group } & \multicolumn{7}{|c|}{ Time of examinations (in months from baseline) } \\
\hline & 2 & 6 & 10 & 14 & 18 & 22 & 26 \\
\hline with B & $\begin{array}{l}74 \pm 1 \\
(47)\end{array}$ & $\begin{array}{l}72 \pm 1 \\
(50)\end{array}$ & $\begin{array}{l}71 \pm 1 \\
(47)\end{array}$ & $\begin{array}{l}71 \pm 1 \\
(47)\end{array}$ & $\begin{array}{l}72 \pm 1 \\
(44)\end{array}$ & $\begin{array}{l}71 \pm 1 \\
(44)\end{array}$ & $\begin{array}{l}70 \pm 1 \\
(45)\end{array}$ \\
\hline without B & $\begin{array}{l}76 \pm 1 \\
(42)\end{array}$ & $\begin{array}{l}76 \pm 1 \\
(46)\end{array}$ & $\begin{array}{l}76 \pm 1 \\
(44)\end{array}$ & $\begin{array}{l}74 \pm 1 \\
(47)\end{array}$ & $\begin{array}{l}74 \pm 1 \\
(39)\end{array}$ & $\begin{array}{l}75 \pm 1 \\
(42)\end{array}$ & $\begin{array}{l}74 \pm 1 \\
(40)\end{array}$ \\
\hline significance & NS & $\mathrm{p}<0.05$ & $\mathrm{p}<0.05$ & $\mathrm{p}<0.05$ & NS & $\mathrm{p}<0.05$ & $\mathrm{p}<0.05$ \\
\hline with $\mathrm{S}$ & $\begin{array}{l}75 \pm 1 \\
(41)\end{array}$ & $\begin{array}{l}74 \pm 1 \\
(48)\end{array}$ & $\begin{array}{l}73 \pm 1 \\
(46)\end{array}$ & $\begin{array}{l}73 \pm 1 \\
(44)\end{array}$ & $\begin{array}{l}73 \pm 1 \\
(39)\end{array}$ & $\begin{array}{l}72 \pm 1 \\
(43)\end{array}$ & $\begin{array}{l}73 \pm 1 \\
(43)\end{array}$ \\
\hline without $S$ & $\begin{array}{l}75 \pm 1 \\
(48)\end{array}$ & $\begin{array}{l}74 \pm 1 \\
(48)\end{array}$ & $\begin{array}{l}73 \pm 1 \\
(45)\end{array}$ & $\begin{array}{l}72 \pm 1 \\
(50)\end{array}$ & $\begin{array}{l}73 \pm 1 \\
(44)\end{array}$ & $\begin{array}{l}73 \pm 1 \\
(43)\end{array}$ & $\begin{array}{l}72 \pm 1 \\
(42)\end{array}$ \\
\hline significance & NS & NS & NS & NS & NS & NS & NS \\
\hline
\end{tabular}

Table 5. Blood glucose values $(\mathrm{mg} / 100 \mathrm{ml})$ at the successive $5 \mathrm{~h}$ OGTT in the 4 treatment groups: placebo (P), biguanide (B), sulphonylurea $(S)$ and combined therapy $(B+S)$. Values represent mean $\pm S E M$

\begin{tabular}{|c|c|c|c|c|c|c|c|c|c|}
\hline \multirow[t]{2}{*}{ Group } & \multirow[t]{2}{*}{$\mathrm{n}$} & \multicolumn{8}{|c|}{ Time (min) after glucose } \\
\hline & & 0 & 15 & 30 & 60 & 120 & 180 & 240 & 300 \\
\hline \multicolumn{10}{|c|}{ a) at 2 months from baseline } \\
\hline $\mathrm{P}$ & 31 & $97 \pm 2$ & $119 \pm 3$ & $155 \pm 5$ & $174 \pm 6$ & $113 \pm 5$ & $73 \pm 3$ & $74 \pm 2$ & $77 \pm 2$ \\
\hline B & 30 & $96 \pm 2$ & $119 \pm 3$ & $155 \pm 4$ & $173 \pm 6$ & $128 \pm 6$ & $77 \pm 4$ & $71 \pm 2$ & $78 \pm 2$ \\
\hline S & 27 & $94 \pm 2$ & $116 \pm 4$ & $157 \pm 6$ & $176 \pm 8$ & $128 \pm 6$ & $73 \pm 3$ & $68 \pm 3$ & $72 \pm 2$ \\
\hline $\mathrm{B}+\mathrm{S}$ & 28 & $93 \pm 3$ & $118 \pm 4$ & $153 \pm 6$ & $171 \pm 7$ & $118 \pm 6$ & $72 \pm 4$ & $69 \pm 2$ & $76 \pm 2$ \\
\hline \multicolumn{10}{|c|}{ b) at 14 months from baseline } \\
\hline $\mathrm{P}$ & 26 & $97 \pm 2$ & $129 \pm 5$ & $160 \pm 6$ & $178 \pm 7$ & $123 \pm 7$ & $79 \pm 5$ & $73 \pm 2$ & $75 \pm 2$ \\
\hline$B$ & 24 & $92 \pm 3$ & $117 \pm 5$ & $153 \pm 6$ & $167 \pm 7$ & $119 \pm 6$ & $71 \pm 4$ & $71 \pm 2$ & $77 \pm 2$ \\
\hline $\mathrm{S}$ & 22 & $92 \pm 3$ & $119 \pm 5$ & $152 \pm 5$ & $165 \pm 8$ & $123 \pm 6$ & $66 \pm 3$ & $66 \pm 3$ & $72 \pm 3$ \\
\hline $\mathrm{B}+\mathrm{S}$ & 24 & $87 \pm 3$ & $117 \pm 5$ & $147 \pm 6$ & $154 \pm 8$ & $107 \pm 4$ & $66 \pm 3$ & $65 \pm 2$ & $73 \pm 2$ \\
\hline \multicolumn{10}{|c|}{ c) at 26 months from baseline } \\
\hline $\mathrm{P}$ & 19 & $95 \pm 2$ & $125 \pm 5$ & $166 \pm 6$ & $188 \pm 9$ & $113 \pm 5$ & $72 \pm 4$ & $73 \pm 3$ & $78 \pm 2$ \\
\hline $\mathrm{B}$ & 23 & $95 \pm 2$ & $128 \pm 5$ & $167 \pm 7$ & $177 \pm 6$ & $115 \pm 5$ & $73 \pm 4$ & $72 \pm 2$ & $79 \pm 2$ \\
\hline$S$ & 22 & $90 \pm 2$ & $114 \pm 4$ & $148 \pm 6$ & $164 \pm 8$ & $113 \pm 6$ & $71 \pm 5$ & $70 \pm 2$ & $74 \pm 3$ \\
\hline$B+S$ & 22 & $93 \pm 3$ & $127 \pm 4$ & $167 \pm 5$ & $176 \pm 9$ & $115 \pm 4$ & $70 \pm 4$ & $69 \pm 3$ & $78 \pm 2$ \\
\hline
\end{tabular}

difference, however, was observed between the $\mathrm{S}$ and $\mathrm{B}+\mathrm{S}$ groups when compared to $\mathrm{B}$ and $\mathrm{P}$ groups.

\section{Blood Glucose}

Mean values of blood glucose levels at the successive $5 \mathrm{~h}$ OGTT are indicated in Tables 5 and 6. No significant effect of dimethylbiguanide could be detected at any time in the course of the trial. In the groups receiving glibenclamide $(\mathrm{S}$ and $\mathrm{B}+\mathrm{S}$ ), blood glucose mean values were generally lower at the 14 months examination than in the groups without $\mathrm{S}(\mathrm{P}$ and B). The 5\% level of significance was reached for observed differences at fasting, 180 and $240 \mathrm{~min}$ after glucose. But at the 2 and 26 months examinations, there was no significant difference.

\section{Plasma Insulin}

Tables 7 and 8 show the failure of glibenclamide and dimethylbiguanide to modify plasma insulin level under the test conditions.

\section{Insulin Glucose Relationships}

They were evaluated by the slope of insulin on glucose regression line calculated for each individual OGTT [12]. Sulphonylurea and biguanide had no apparent effect on these relationships (Table 9). 
Table 6. Effect of biguanide (B) and sulphonylurea (S) on blood glucose values $(\mathrm{mg} / 100 \mathrm{ml})$ at the successive $5 \mathrm{~h}$ OGTT. Values represent mean \pm SEM. $p$ values are calculated by a two way analysis of variance

\begin{tabular}{|c|c|c|c|c|c|c|c|c|c|}
\hline \multirow[t]{2}{*}{ Groups } & \multirow[t]{2}{*}{$\mathrm{n}$} & \multicolumn{8}{|c|}{ Time (min) after glucose } \\
\hline & & 0 & 15 & 30 & 60 & 120 & 180 & 240 & 300 \\
\hline \multicolumn{10}{|c|}{ a) at 2 months from baseline } \\
\hline with B & 58 & $95 \pm 2$ & $118 \pm 6$ & $154 \pm 4$ & $172 \pm 5$ & $123 \pm 4$ & $74 \pm 3$ & $70 \pm 2$ & $77 \pm 2$ \\
\hline $\begin{array}{l}\text { without B } \\
\text { significance }\end{array}$ & 58 & $96 \pm 2$ & $117 \pm 6$ & $156 \pm 4$ & $175 \pm 5$ & $120 \pm 4$ & $73 \pm 2$ & $72 \pm 2$ & $75 \pm 2$ \\
\hline with $S$ & 55 & $94 \pm 2$ & $117 \pm 3$ & $155 \pm 4$ & $173 \pm 5$ & $123 \pm 4$ & $73 \pm 5$ & $69 \pm 2$ & $74 \pm 1$ \\
\hline $\begin{array}{l}\text { without } S \\
\text { significance }\end{array}$ & 61 & $97 \pm 2$ & $119 \pm 2$ & $155 \pm 4$ & $173 \pm 5$ & $120 \pm 4$ & $75 \pm 2$ & $73 \pm 1$ & $77 \pm 1$ \\
\hline \multicolumn{10}{|c|}{ b) at 14 months from baseline } \\
\hline with B & 48 & $89 \pm 2$ & $117 \pm 3$ & $157 \pm 4$ & $160 \pm 5$ & $113 \pm 4$ & $69 \pm 3$ & $68 \pm 2$ & $75 \pm 2$ \\
\hline $\begin{array}{l}\text { without B } \\
\text { significance }\end{array}$ & 48 & $95 \pm 2$ & $124 \pm 3$ & $156 \pm 4$ & $172 \pm 5$ & $123 \pm 4$ & $73 \pm 3$ & $70 \pm 2$ & $73 \pm 2$ \\
\hline with S & 46 & $89 \pm 2$ & $118 \pm 3$ & $149 \pm 4$ & $159 \pm 5$ & $114 \pm 4$ & $66 \pm 3$ & $70 \pm 2$ & $72 \pm 2$ \\
\hline $\begin{array}{l}\text { without } S \\
\text { significance }\end{array}$ & 50 & $\begin{array}{l}95 \pm 2 \\
p<0.05\end{array}$ & $124 \pm 3$ & $157 \pm 4$ & $173 \pm 5$ & $121 \pm 4$ & $\begin{array}{l}75 \pm 3 \\
p<0.05\end{array}$ & $\begin{array}{l}72 \pm 2 \\
p<0.05\end{array}$ & $76 \pm 2$ \\
\hline \multicolumn{10}{|c|}{ c) at 26 months from baseline } \\
\hline with B & 45 & $94 \pm 2$ & $127 \pm 3$ & $167 \pm 4$ & $176 \pm 5$ & $115 \pm 4$ & $71 \pm 3$ & $71 \pm 2$ & $78 \pm 1$ \\
\hline $\begin{array}{l}\text { without B } \\
\text { significance }\end{array}$ & 41 & $93 \pm 2$ & $119 \pm 3$ & $156 \pm 4$ & $175 \pm 6$ & $113 \pm 4$ & $71 \pm 3$ & $71 \pm 2$ & $76 \pm 2$ \\
\hline with S & 44 & $92 \pm 2$ & $120 \pm 3$ & $157 \pm 4$ & $170 \pm 6$ & $114 \pm 4$ & $71 \pm 3$ & $69 \pm 2$ & $76 \pm 2$ \\
\hline $\begin{array}{l}\text { without S } \\
\text { significance }\end{array}$ & 42 & $95 \pm 2$ & $127 \pm 3$ & $166 \pm 4$ & $182 \pm 6$ & $114 \pm 4$ & $72 \pm 3$ & $73 \pm 2$ & $78 \pm 2$ \\
\hline
\end{tabular}

Table 7. Plasma insulin values $(\mu \mathrm{U} / \mathrm{ml})$ at the successive 5 h OGTT in the 4 groups of treatment: placebo (P), biguanide (B), sulphonylurea $(S)$ and combined therapy $(B+S)$. Values represent mean $\pm S E M$

\begin{tabular}{|c|c|c|c|c|c|c|c|c|c|c|}
\hline \multirow[t]{2}{*}{ Group } & \multirow[t]{2}{*}{$\mathrm{n}$} & \multicolumn{9}{|c|}{ Time (min) after glucose } \\
\hline & & 0 & 15 & 30 & 60 & 120 & & 180 & 240 & 300 \\
\hline \multicolumn{11}{|c|}{ a) at 2 months from baseline } \\
\hline $\mathrm{P}$ & 30 & $16 \pm 1$ & $40 \pm 4$ & $64 \pm 10$ & $94 \pm 11$ & $65 \pm$ & 8 & $21 \pm 2$ & $14 \pm 1$ & $13 \pm 1$ \\
\hline B & 27 & $13 \pm 1$ & $36 \pm 3$ & $58 \pm 7$ & $76 \pm 10$ & $65 \pm$ & 8 & $23 \pm 3$ & $10 \pm 1$ & $10 \pm 1$ \\
\hline S & 24 & $15 \pm 2$ & $32 \pm 3$ & $49 \pm 7$ & $64 \pm 7$ & $56 \pm$ & 8 & $25 \pm 5$ & $11 \pm 1$ & $10 \pm 1$ \\
\hline$B+S$ & 25 & $13 \pm 1$ & $36 \pm 5$ & $47 \pm 6$ & $82 \pm 9$ & $58 \pm$ & 7 & $26 \pm 5$ & $13 \pm 1$ & $12 \pm 1$ \\
\hline
\end{tabular}

b) at 14 months from baseline

\begin{tabular}{|c|c|c|c|c|c|c|c|c|c|}
\hline $\mathrm{P}$ & 26 & $16 \pm 1$ & $41 \pm 5$ & $62 \pm 8$ & $76 \pm 6$ & $57 \pm 5$ & $23 \pm 3$ & $15 \pm 1$ & $13 \pm 1$ \\
\hline $\mathrm{B}$ & 23 & $12 \pm 1$ & $32 \pm 4$ & $59 \pm 6$ & $86 \pm 10$ & $57 \pm 7$ & $19 \pm 3$ & $11 \pm 2$ & $13 \pm 3$ \\
\hline S & 19 & $15 \pm 2$ & $46 \pm 10$ & $65 \pm 12$ & $72 \pm 10$ & $77 \pm 14$ & $19 \pm 3$ & $12 \pm 2$ & $11 \pm 2$ \\
\hline$B+S$ & 24 & $13 \pm 2$ & $37 \pm 4$ & $47 \pm 5$ & $66 \pm 7$ & $54 \pm 7$ & $21 \pm 4$ & $11 \pm 1$ & $10 \pm 1$ \\
\hline
\end{tabular}

c) at 26 months from baseline

\begin{tabular}{llllllllll}
$\mathrm{P}$ & 19 & $16 \pm 2$ & $34 \pm 5$ & $53 \pm 6$ & $75 \pm 7$ & $61 \pm 10$ & $19 \pm 3$ & $13 \pm 2$ & $11 \pm 1$ \\
$\mathrm{~B}$ & 23 & $13 \pm 1$ & $30 \pm 5$ & $55 \pm 7$ & $82 \pm 10$ & $58 \pm 8$ & $19 \pm 3$ & $11 \pm 1$ & $11 \pm 1$ \\
$\mathrm{~S}$ & 21 & $15 \pm 2$ & $31 \pm 5$ & $48 \pm 7$ & $69 \pm 10$ & $55 \pm 8$ & $20 \pm 4$ & $11 \pm 1$ & $10 \pm 1$ \\
$\mathrm{~B}+\mathrm{S}$ & 21 & $10 \pm 1$ & $34 \pm 5$ & $51 \pm 8$ & $60 \pm 8$ & $44 \pm 6$ & $14 \pm 5$ & $10 \pm 1$ & $9 \pm 1$ \\
\hline
\end{tabular}

\section{Efficiency of the Experiment}

In order to investigate if the absence of significant effect of treatments on blood glucose could be due to a lack of power of the study test, theoretical differ- ences which would have been significant with a probability equal to 0.95 were calculated. An example of these minimal differences is indicated for 2 months in table 10. They are small enough to be reasonably expected if the therapies used were minimally efficient. 
Table 8. Effect of biguanide (B) and sulphonylurea (S) on plasma insulin values $(\mu \mathrm{U} / \mathrm{ml})$ at the successive $5 \mathrm{~h}$ OGTT. Values represent mean \pm SEM. p values are calculated by a two any analysis of variance

\begin{tabular}{|c|c|c|c|c|c|c|c|c|c|}
\hline \multirow[t]{2}{*}{ Groups } & \multirow[t]{2}{*}{$\mathrm{n}$} & \multicolumn{8}{|c|}{ Time (min) after glucose } \\
\hline & & 0 & 15 & 30 & 60 & 120 & 180 & 240 & 300 \\
\hline \multicolumn{10}{|c|}{ a) at 2 months from baseline } \\
\hline with $\mathrm{B}$ & 52 & $13 \pm 1$ & $36 \pm 3$ & $53 \pm 5$ & $79 \pm 7$ & $62 \pm 6$ & $24 \pm 3$ & $12 \pm 1$ & $11 \pm 1$ \\
\hline $\begin{array}{l}\text { without B } \\
\text { significance }\end{array}$ & 54 & $15 \pm 1$ & $36 \pm 3$ & $57 \pm 5$ & $80 \pm 7$ & $61 \pm 5$ & $23 \pm 3$ & $13 \pm 1$ & $11 \pm 1$ \\
\hline with S & 49 & $14 \pm 1$ & $34 \pm 3$ & $48 \pm 6$ & $73 \pm 7$ & $57 \pm 6$ & $25 \pm 3$ & $12 \pm 1$ & $11 \pm 1$ \\
\hline $\begin{array}{l}\text { without } S \\
\text { significance }\end{array}$ & 57 & $14 \pm 1$ & $38 \pm 3$ & $62 \pm 5$ & $85 \pm 7$ & $65 \pm 5$ & $22 \pm 3$ & $12 \pm 1$ & $11 \pm 1$ \\
\hline \multicolumn{10}{|c|}{ b) at 14 months from baseline } \\
\hline with $\mathbf{B}$ & 47 & $12 \pm 1$ & $35 \pm 4$ & $53 \pm 6$ & $75 \pm 6$ & $56 \pm 6$ & $20 \pm 2$ & $11 \pm 1$ & $11 \pm 1$ \\
\hline $\begin{array}{l}\text { without B } \\
\text { significance }\end{array}$ & 45 & $\begin{array}{l}16 \pm 1 \\
p<0.05\end{array}$ & $43 \pm 4$ & $64 \pm 6$ & $74 \pm 6$ & $66 \pm 6$ & $22 \pm 2$ & $14 \pm 1$ & $12 \pm 1$ \\
\hline with S & 43 & $14 \pm 1$ & $41 \pm 4$ & $55 \pm 6$ & $69 \pm 6$ & $64 \pm 6$ & $20 \pm 2$ & $12 \pm 1$ & $10 \pm 1$ \\
\hline $\begin{array}{l}\text { without } S \\
\text { significance }\end{array}$ & 49 & $14 \pm 1$ & $37 \pm 4$ & $61 \pm 6$ & $80 \pm 6$ & $57 \pm 6$ & $21 \pm 2$ & $13 \pm 1$ & $13 \pm 1$ \\
\hline \multicolumn{10}{|c|}{ c) at 26 months from baseline } \\
\hline with B & 44 & $11 \pm 1$ & $32 \pm 3$ & $54 \pm 5$ & $72 \pm 6$ & $51 \pm 5$ & $17 \pm 2$ & $11 \pm 1$ & $10 \pm 1$ \\
\hline $\begin{array}{l}\text { without B } \\
\text { significance }\end{array}$ & 40 & $15 \pm 1$ & $33 \pm 3$ & $51 \pm 5$ & $72 \pm 7$ & $58 \pm 6$ & $20 \pm 2$ & $12 \pm 1$ & $10 \pm 1$ \\
\hline with $\mathrm{S}$ & 42 & $12 \pm 1$ & $32 \pm 3$ & $50 \pm 5$ & $65 \pm 6$ & $49 \pm 6$ & $17 \pm 2$ & $11 \pm 1$ & $9 \pm 1$ \\
\hline $\begin{array}{l}\text { without S } \\
\text { significance }\end{array}$ & 42 & $14 \pm 1$ & $33 \pm 3$ & $54 \pm 4$ & $79 \pm 6$ & $59 \pm 6$ & $19 \pm 2$ & $12 \pm 1$ & $11 \pm 1$ \\
\hline
\end{tabular}

Table 9. Effect of biguanide (B) and sulphonylurea (S) on the slope of $I$ on $G$ regression line

\begin{tabular}{|c|c|c|c|c|c|c|c|c|}
\hline & \multirow[b]{2}{*}{$\mathrm{n}$} & \multicolumn{7}{|c|}{ Time of examination (in months from baseline) } \\
\hline & & $\begin{array}{l}0 \\
\mathrm{~m} \pm \mathrm{SEM}\end{array}$ & $\mathbf{n}$ & $\begin{array}{l}2 \\
\mathrm{~m} \pm \mathrm{SEM}\end{array}$ & $\mathrm{n}$ & $\begin{array}{l}14 \\
\mathrm{~m} \pm \mathrm{SEM}\end{array}$ & $\mathrm{n}$ & $\begin{array}{l}26 \\
\mathrm{~m} \pm \mathrm{SEM}\end{array}$ \\
\hline $\mathrm{P}$ & 33 & $0.59 \pm 0.09$ & 29 & $0.76 \pm 0.11$ & 25 & $0.53 \pm 0.06$ & 19 & $0.50 \pm 0.06$ \\
\hline $\mathrm{B}$ & 30 & $0.66 \pm 0.07$ & 26 & $0.66 \pm 0.07$ & 24 & $0.69 \pm 0.07$ & 22 & $0.62 \pm 0.10$ \\
\hline$S$ & 28 & $0.56 \pm 0.06$ & 24 & $0.55 \pm 0.10$ & 19 & $0.75 \pm 0.16$ & 21 & $0.52 \pm 0.08$ \\
\hline $\mathrm{B}+\mathrm{S}$ & 29 & $0.60 \pm 0.08$ & 25 & $0.64 \pm 0.07$ & 24 & $0.59 \pm 0.08$ & 20 & $0.55 \pm 0.11$ \\
\hline with B & 59 & $0.63 \pm 0.06$ & 51 & $0.65 \pm 0.07$ & 48 & $0.64 \pm 0.06$ & 42 & $0.59 \pm 0.06$ \\
\hline $\begin{array}{l}\text { without } B \\
p \text { value }\end{array}$ & 61 & $0.58 \pm 0.06$ & 53 & $0.67 \pm 0.07$ & 44 & $0.63 \pm 0.07$ & 40 & $0.51 \pm 0.06$ \\
\hline with S & 57 & $0.58 \pm 0.06$ & 49 & $0.60 \pm 0.07$ & 43 & $0.66 \pm 0.07$ & 41 & $0.53 \pm 0.06$ \\
\hline $\begin{array}{l}\text { without } S \\
\mathrm{p} \text { value }\end{array}$ & 63 & $0.63 \pm 0.06$ & 55 & $0.71 \pm 0.06$ & 49 & $0.61 \pm 0.06$ & 42 & $0.57 \pm 0.06$ \\
\hline
\end{tabular}

\section{Discussion}

In the present study, sulphonylurea had no effect even an adverse effect - on weight reduction, while administration of biguanide is accompanied by weight reduction, in accordance with the results reported by Feldman [24] and Clarke [36]. Blood glucose improvement was only observed in the $\mathrm{S}$ group at the 14 months test. A significant level was reached only at fasting and at 180 and $240 \mathrm{~min}$ after oral glucose. Under the null hypothesis (i.e. no effect) the probability of observing three significant differences of identical sign among the 48 involved comparisons was equal to $11 \%$. Under the alternative hypothesis (i. e. efficacy of drugs as defined in table 10), the probability of obtaining only three significant differences was near zero. So there was no evidence of the effectiveness of oral hypoglycaemic drugs either in lowering blood glucose or in improving insulin secretion in borderline diabetic patients. These results are also in agreement with Feldman's study [24]. However, some differences which might 
Table 10. Lower limits of differences of blood glucose values which would have been detected with a probability of $95 \%$ when groups with $\mathrm{S}$ are compared to groups without $\mathrm{S}-$ or groups with $\mathrm{B}$ are compared to groups without B

\begin{tabular}{|c|c|c|c|c|c|c|c|}
\hline & \multicolumn{7}{|c|}{ Time (min) after glucose load } \\
\hline & 0 & 15 & $30 \quad 60$ & 120 & 180 & 240 & 300 \\
\hline $\begin{array}{l}\text { Differences in } \\
\text { blood glucose } \\
\text { levels } \\
(\mathrm{mg} / 100 \mathrm{ml})\end{array}$ & 8 & 12.5 & $19 \quad 27$ & 21 & 12 & 7 & 7 \\
\hline \multicolumn{8}{|c|}{$\begin{array}{l}\text { These values are given to appreciate the efficiency of the study } \\
\text { For instance, let be } 21 \mathrm{mg} / 100 \mathrm{ml} \text { the true difference (unknown } \\
\text { due to treatment at } 120 \mathrm{~min} \text { after glucose. In this study, with th } \\
\text { number of subjects examinated at } 2 \text { months, the probability th } \\
\text { observe a significant difference was equal to } 95 \% \text {. In the sam } \\
\text { conditions, a true difference equal to } 11 \mathrm{mg} / 100 \mathrm{ml} \text { had only } \\
\text { probability of } 50 \% \text { to be detected }\end{array}$} \\
\hline
\end{tabular}

have interfered with the results are notable between these two trials. Among them are the different patterns of drug intake before the test: in Feldman's study treatments were discontinued 3 days before each OGTT showing the absence of a permanent effect of drugs on glucose tolerance. In the present study, drugs were not discontinued before the tests except before the last test. Nevertheless no striking effectiveness of the drugs in lowering blood glucose was shown, although the overall procedure of the trial was accurate enough to disclose small differences between the groups. This is of interest because the doses of drugs we used were similar to those usually given in asymptomatic diabetes.

In the present study, a sulphonylurea-biguanide association previously advocated [25] could be tested and was found to lack totally any synergic effect on blood glucose. There was no evidence of a significant effect of drugs on insulin levels and the insulin-glucose relationship evaluated by an index currently used [26-27] was not altered by drugs. Thus, in a long-term experiment, biguanide was not able to diminish plasma insulin level, and sulphonylurea had no apparent stimulatory effect on insulin secretion. This failure of sulphonylurea to increase insulin secretion under chronic administration has been previously demonstrated by $\mathrm{CHU}$ et al. [28] and emphasized by PROUT [29].

This study shows that there is no clear beneficial effect of oral hypoglycaemic drugs on blood glucose levels in borderline diabetics confirming previous findings $[37,24]$. In view of the disturbing UGDP [30-32] findings which suggested a possible toxic effect of oral hypoglycaemic drugs, our results support the concept that there is no rational basis for the use of those agents in patients with mild impairment of glucose tolerance [33].

Acknowledgements. We thank A. Fenelon for her invaluable technical assistance. The study was supported by grants from I.N.S.E.R.M.

\section{References}

1. Pirart, J.: Diabete et complications dégénératives. Présentation d'une étude prospective portant sur 4400 cas observés entre 1947 et 1973. I. Diabéte Métab. 3, 97-107 (1977)

2. Pirart, J.: Diabete et complications dégénératives. Présentation d'une étude prospective portant sur 4400 cas observés entre 1947 et 1973. II. Diabéte Métab. 3, 173-182 (1977)

3. Job, D., Eschwege, E., Guyot-Argenton, C., Aubry, J.P., Tchobroutsky, G.: Effect of multiple daily insulin injections on the course of diabetic retinopathy. Diabetes 25, 463-469 (1976)

4. Engerman, R. L.: Pathophysiology of diabetic retinopathy, $p$ 194. Report of the national commission on Diabetes, Vol. 3 , part 3. Dhew Publication No. (NIH) 76-1023. Washington, DC: Government Printing Office 1976

5. Hagg, E.: On the pathogenesis of glomerular lesions in the alloxen diabetic rat. Acta Med. Scand. [Suppl.] 558, 1-31 (1974)

6. Mauer, S. M., Sutherland, D. E. R., Steffes, M. W., Leonard, R. J., Najarian, J. S., Michael, A. F., Brown, D. F.: Pancreatic islet transplantation. Diabetes 23, 748-753 (1974)

7. Eschwege, E.: Short term effects ( 2 years) of oral treatment for diabetes on the borderline impairment of oral glucose tolerance test. Diabetologia 10, 363 (1974)

8. Valleron, A.J., Aboulker, J. P., Papoz, L., Rathery, M. Reproducibility of borderline $0-2$ hrs OGTT. In: Diabetes epidemiology in Europe. H. Gutsche, H.-D. Holler (Ed.), p 109. Stuttgart: Thieme 1975

9. McDonald, G. W., Fisher, G. F., Burnham, C.: Reproducibility of the oral glucose tolerance test. Diabetes 14, 473-480 (1965)

10. McDonald, G. W., Burnham, C., Lewis, W. F.: Reproducibility of glucose tolerance in 101 non-diabetic women. Public Health Rep. 79, 353 (1969)

11. Beck, H., Job, D., Sebban, C., Vignalou, J.: Reproductibilité de la mesure de la glycémie chez le sujet âgé. Geront. Clin. 15, 37-44 (1973)

12. Job, D., Papoz, L., Eschwege, E., Rosselin, G. E.: Reproducibility of the glucose and insulin response to identical oral glucose load performed one year apart. Diabete Métab. 4, 95-101 (1978)

13. Hugget, A., Nixon, D. A.: Use of glucose oxidase peroxidase or $\mathrm{O}$ - dianisidine in the determination of blood and urine glucose. Lancet 1957 II, 368

14. European Association for the Study of Diabetes: A Brief Account of the European Diabetes Epidemiology Study Group Recommendation and Research. Diabetologia 6, 453-454 (1970)

15. Gutsche, H., Holler, H. D.: Diabetes epidemiology in Europe, p. 197. Stuttgart: Thieme 1975

16. Committee on Statistics on the American Diabetes Association: Standardization of the oral glucose tolerance test. Diabetes 18, 299-310 (1969)

17. Eschwege, E., Valleron, A. J., Papoz, L., Pequignot, G., Rosselin, G. E.: Classification and choice of the blood glucose values during oral glucose tolerance test as selection criteria for clinical trial in diabetes prevention. Proceedings of the 
International Symposium on Diabetes Sofia 23-25, November 1970 , p. 23. Publishing house of the Bulgarian Academy of Sciences 1972

18. Wilkerson, H. L. C.: Diagnosis oral glucose tolerance test, p. 31. In: Diabetes Mellitus - Diagnosis and Treatment. New York: American Diabetes Association 1964

19. Fitzgerald, M. G., Keen, H.: Diagnostic classification of diabetes. Br. Med. J. 1964 I, 1586

20. Valleron, A. J., Eschwege, E., Papoz, L., Rosselin, G. E.: Agreement and discrepancy in the evaluation of normal and diabetic oral glucose tolerance test. Diabetes 24, 585-593 (1975)

21. Documenta Geigy: Naturwissenschaftliche Tabellen, 6th Ed., p. 634. Basel: Jr Geigy, AG 1963

22. Rosselin, G. E., Dolais, J.: Application de la methode radio immunologique du dosage de l'insuline humaine et du dosage de l'hormone folliculo-stimulante humaine (HFSH), p. 189-217. Les isotopes. Applications biocliniques. Monographie annuelle de la Société Française de Biologie Clinique, 1967. Paris: Expansion Scientifique Française 1967

23. Lellouch, J., Lazar, P.: Méthodes statistiques en expérimentation biologique, p. 165. Paris: Flammarion médecine-sciences 1974

24. Feldman, R., Crawford, D., Elashoff, R., Glass, A.: Oral Hypoglycemic drugs prophylaxis in asymptomatic diabetes. In: Diabetes, proceedings of the 8 th congress of the IDF. W. J. Malaisse, J. Pirart, J. Vallance-Owen (Ed.), p. 574. Amsterdam: Excerpta Medica; New York: American Elsevier Publishing Company, Inc. 1974

25. Mehnert, $\mathrm{H}$.: The biguanides: mechanism of action and clinical application. In: Diabetes, proceedings of the 8th congress of the IDF. W. J. Malaisse, J. Pirart, J. Vallance-Owen (Ed.), p. 603. Amsterdam: Excerpta Medica; New York: American Elsevier Publishing Company, Inc. 1974

26. Soeldner, J. S., Gleason, R. E., Williams, R. F., Garcia, M. J., Bearwood, D. M., Marble, A.: Diminished serum insulin response to glucose in genetic prediabetic males with normal glucose tolerance. Diabetes 17, 17-26 (1968)

27. Colwell, J.A., Lein, A.: Diminished insulin response to hyperglycemia in prediabetes and diabetes. Diabetes 16, 560-565 (1967)

28. Chu, P., Conway, M. J., Krouse, H. A., Goodner, C. J.: The pattern of response of plasma insulin and glucose to meals and fasting during chlorpropamide therapy. Ann. Intern. Med. 68, 757 (1968)
29. Prout, T. E.: Adverse effects of oral hypoglycemic drugs. In: Diabetes, Proceedings of the 8 th congress of IDF. W. J. Malaisse, J. Pirart, J. Vallance-Owen (Ed.), p.612. Amsterdam: Excerpta Medica; New York: American Elsevier Publishing Company, Inc. 1974

30. Klimt, C. R., Meinert, C. L., Miller, C. L., Knowles, H. C.: UGDP, A study of relationships of therapy to vascular and other complications of diabetes. In: Tolbutamide after ten years, p. 261. Brook/Lodge symposium, August 1967. New York: Excerpta Medica Found. Int. Congr. Series No. 149 1967

31. UGDP: I Design, methods and baseline characteristics - II Mortality results. Diabetes 19 (Suppl. 2), 747-789 (1970)

32. UGDP: V Evaluation of phenformin therapy. Diabetes $\mathbf{2 4}$ (Suppl. 1), 65-184 (1974)

33. British Diabetes Association: Diagnostic criteria for diabetes mellitus. Recommendations, Medical and Scientific section, April 1978

34. Fajans, S. S., Conn, J. S.: Prediabetes, subclinical diabetes and latent clinical diabetes. Interpretation, diagnosis and treatment. In: on the Nature and Treatment of Diabetes, p. 641. New York: Excerpta Medica Found. Int. Congr. Series No. 84 1965

35. McDonald, G. W., Hoet, J. P., Butterfield, W. J. H.: Diabetes Mellitus: Report of WHO expert commitee. WHO Rep. Series No. 310 (1965)

36. Clarke, B. F., Campbell, I. W.: Comparison of metformin and chlorpropamide in non-obese, maturity-onset diabetics uncontrolled by diet. Br. Med. J. 1977 II, 1576-1578

37. Keen, H., Jarrett, R. J., Fuller, J. H.: Tolbutamide and arterial disease in borderline diabetics. In: Proceedings of 8th Congress of the Int. Diab. Fed, W. J. Malaisse, J. Pirart (Eds.), p. 588. Amsterdam: Excerpta Medica 1974

Received: February 13, 1978,

and in revised form: June 26, 1978

Laure Papoz, M. A.

INSERM

Unité de Recherches Statistiques

16 bis avenue Paul Vaillant Couturier

F-94800 Villejuif

France 